\title{
AN AXIOMATIC AND NON-COOPERATIVE APPROACH TO THE MULTI-STEP SHAPLEY VALUE
}

\author{
XiAnghui Li ${ }^{1, *}$, Wei Zheng ${ }^{2}$ AND YANG $\mathrm{LI}^{3}$
}

\begin{abstract}
Inspired by the two-step Shapley value, in this paper we introduce and axiomatize the multi-step Shapley value for cooperative games with levels structures. Moreover, we design a multistep bidding mechanism, which implements the value strategically in subgame perfect Nash equilibrium for superadditve games.
\end{abstract}

Mathematics Subject Classification. 91A10, 91A12.

Received September 7, 2020. Accepted April 30, 2021.

\section{INTRODUCTION}

Many realistic situations can be modeled as cooperative games. A cooperative game consists of a finite player set and a characteristic function which assigns to each coalition, i.e., a subset of the player set, a real number, representing the aggregate benefit of players in it from cooperation. A central problem in the cooperative game theory is how to distribute the gains of cooperation among all players in a reasonable way. One of the most widely used single-valued solutions to this problem is the Shapley value [13].

Considering that players may partition themselves into disjoint coalitions before cooperation, Aumann and Dreze [1] introduced a model of coalition structure. Once the coalition structure is determined, negotiations can take place only within each of the coalitions that constitute the structure. Many solutions have been proposed for this type of games with coalition structure, the most well known of which are the Owen value [11] and the Aumann and Dreze value [1]. Later, Kamijo [7] proposed the two-step Shapley value. All of these values are extensions of the Shapley value.

However, in many practical situations, a coalition structure can not adequately describe the external cooperation between the coalitions themselves, which might also be relevant for deciding the payoff distribution between the players. Owen [11] generalized the coalition structure by considering these cases and introduced a model of levels structure. A levels structure is a sequence of coalition structures, where each is obtained from the previous one by unification of coalitions and represents the various forms of cooperation between coalitions of the previous coalition structure. A levels structure value was also proposed as a generalization of the Owen value.

Keywords. Multi-step shapley value, levels structure, implementation, SPNE.

1 Department of Mathematics, School of Sciences, Henan University of Technology, Zhengzhou 450001, Henan, PR China.

2 School of Mathematics and Statistics, Shandong Normal University, Ji'nan 250014, PR China.

3 School of Sciences, Henan University of Technology, Zhengzhou 450001, Henan, PR China.

*Corresponding author: xianghuili_1107@163.com 
In this paper, we develop a distributive analysis of cooperative surplus among players when they have organized themselves into a levels structure before realizing cooperation. We define a new solution concept, called a multi-step Shapley value, for cooperative games with levels structures. It can be interpreted as the result of bargaining of the lower-level unions on the worth of the higher-level unions they join. Analogous to the various axiomatizations $[10,13,19]$ of the Shapley value, we provide several axiomatizations of the multi-step Shapley value, too.

After a solution concept for cooperative games has been defined, it seems natural to ask question whether there exists a non-cooperative framework to give rise to this value. Pérez-Castrillo and Wettstein [12] provided a non-cooperative bidding mechanism and showed that the subgame perfect Nash equilibrium $(S P N E)$ outcomes of this mechanism always coincide with the vector of the Shapley value payoffs for zero-monotonic cooperative games.

Later, some variants of the bidding mechanism emerge for implementing other solution concepts. Ju et al. [5] designed a two-level bidding mechanism to implement the consensus value [5]. Ju and Wettstein [6] provided a non-cooperative foundation to several cooperative solution concepts, such as the Shapley value and consensus value, by using a class of bidding mechanisms that differ in the power awarded to the proposer chosen through a bidding process. Van den Brink et al. [15] incorporated the possibility of breakdown of the negotiations into the bidding mechanism in Pérez-Castrillo and Wettstein [12] and showed that the $\alpha$-egalitarian Shapley value defined by Joosten [4] is implemented as the equilibrium payoff. Van den Brink and Funaki [14] introduced discounting in the bidding mechanism and the modified bidding mechanism yields the corresponding discounted Shapley value in Joosten [4] as the payoff distribution in every subgame perfect Nash equilibrium. Vidal-Puga and Bergantiños [16] gave a coalitional bidding mechanism which has two rounds and implements the Owen value. Vidal-Puga [17] proposed a levels bidding mechanism which is played in several rounds and implements the levels structure value. Based on these research, we proceed by establishing a multi-step bidding mechanism such that players behave strategically and obtain as the final outcome the multi-step Shapley value in every subgame perfect Nash equilibrium for superadditive games. In some respects, we provide a new non-cooperative approach to the two-step Shapley value different to Kamijo [8] as well, since the multi-step Shapley value is equal to the two-step Shapley value associated with the induced coalition structure when the levels structure has the degree 2 .

This paper is organized as follows. Section 2 is the basic information about cooperative games and levels structures. In Section 3.1, we define a multi-step Shapley value and give its axiomatizations. In Section 4, we give a multi-step bidding mechanism which implements the multi-step Shapley value for superadditive game. Section 5 concludes.

\section{Preliminaries}

\subsection{Cooperative games}

A cooperative game can be described by a pair $(N, v)$, where $N=\{1,2, \ldots, n\}$ is the set of players and $v: 2^{N} \rightarrow \mathbb{R}$ with $v(\emptyset)=0$ is the corresponding characteristic function. Any subset $S \subseteq N$ is called a coalition and $v(S)$ the worth of $S$. If there is no ambiguity, we identify the game $(N, v)$ with its characteristic function $v$. The set of all cooperative games over $N$ is denoted by $G(N)$. We use the notation $|\cdot|$ to represent the number of members in a set.

The restriction of a game $(N, v)$ to a coalition $S \subseteq N$, denoted by $\left(S, v_{S}\right)$, is defined as $v_{S}(T)=v(T)$ for all $T \subseteq S$. For simplicity, we write $(S, v)$ instead of $\left(S, v_{S}\right)$. A cooperative game $(N, v)$ is called zero-monotonic if $v(S \cup\{i\}) \geq v(S)+v(\{i\})$ for any $S \subseteq N \backslash\{i\}$, and called superadditive if for all $S, T \subseteq N$ such that $S \cap T=\emptyset$, $v(S \cup T) \geq v(S)+v(T)$.

A value for cooperative game $(N, v)$ is a mapping $\phi: G^{n} \rightarrow \mathbb{R}^{n}$. The famous Shapley value for cooperative games is defined as

$$
S h_{i}(N, v)=\sum_{T \subseteq N ; i \in T} \frac{(|N|-|T|) !(|T|-1) !}{|N| !}[v(T)-v(T \backslash\{i\})],
$$


for any $i \in N$.

\subsection{Levels structures}

Aumann and Dreze [1] introduced a simplest model of limited cooperation, coalition structure, which is specified by a partition of the player set.

A collection $\left\{B_{1}, B_{2}, \ldots, B_{m}\right\}$ of subsets of $N$ is called a partition on $N$ iff $B_{1} \cup \cdots \cup B_{m}=N$ and $B_{k} \cap B_{l}=\emptyset$ for $k \neq l, k, l \in\{1,2, \ldots, m\}$.

A coalition structure on $N$ is a partition $B=\left\{B_{1}, B_{2}, \ldots, B_{m}\right\}$ of $N$.

The restriction to $S \subseteq N$ of a coalition structure $B$ is denoted by $B(S)=\left\{B_{k}: B_{k} \subseteq S, k \in\{1,2, \ldots, m\}\right\}$.

For a cooperative game with coalition structure $(N, v, B),\left(M, v^{M}\right)$ is a game among coalitions where $M=$ $\{1,2, \ldots, m\}$ is a set of coalitional indices of the elements in $B$ and $v^{M}(H)=v\left(\bigcup_{k \in H} B_{k}\right)$ for each $H \subseteq M$.

Kamijo [7] defined a solution, called the two-step Shapley value for cooperative games with coalition structures.

Definition 2.1. (Kamijo [7]) A two-step Shapley value $T S(N, v, B)$ for $(N, v, B)$ is defined by

$$
T S_{i}(N, v, B)=S h_{i}\left(B_{k}, v\right)+\frac{S h_{k}\left(M, v^{M}\right)-v\left(B_{k}\right)}{\left|B_{k}\right|}, \quad \forall i \in B_{k} \in B .
$$

The two-step Shapley value allocates the cooperative surplus by using the Shapley value in a two-step bargaining process: one step within coalitions and one between coalitions. Moreover, the bargaining surplus, i.e., $S h_{k}\left(M, v^{M}\right)-v\left(B_{k}\right)$ of each coalition $B_{k}$ is allocated among its members in an egalitarian way.

Winter [18] took into account the cooperation between coalitions and extends games with coalition structure to the so called games with levels structure of cooperation. The levels structure means a sequence of coalition structures, each obtained from the previous by unification of coalitions and each representing the various cooperations between coalitions of the previous coalition structure. Each coalition structure is called a "level".

A levels structure over $N$ of degree $h$ is a sequence of partitions $\mathcal{B}=\left(B^{0}, B^{1}, \ldots, B^{h}\right)$ of $N$ with $B^{0}=$ $\{\{i\} \mid i \in N\}$ such that for any $S \in B^{k}, k \in\{0,1, \ldots, h-1\}$, there is $A \subseteq B^{k-1}$ such that $S=\bigcup_{T \in A} T$.

In this paper, we only consider the case that the grand coalition will eventually form, i.e., $B^{h}=\{N\}$. The set of levels structures on $N$ is denoted by $L S^{N}$. The levels structure $\mathcal{B}$ has $h+1$ levels. $B^{k}$ is called the $k$ th level of $\mathcal{B}$ and each $S \in B^{k}$ is a union at level $k$.

The restriction of $\mathcal{B}$ to a union $U \in B^{k}$ at level $k$ is a levels structure $\mathcal{B}(U)$ on the player set $U$ with $\mathcal{B}(U)=\left(B^{1}(U), B^{2}(U), \ldots, B^{k}(U)\right)$, where $B^{l}(U), \forall l \in\{1,2, \ldots, k\}$ is the restriction to $U$ of coalition structure $B^{l}$. For any $T \in B^{k}$ and $S \subseteq T$, we use the notation $B_{S}^{k}$ to represent the union at level $k$ that contains the subset $S$, i.e., $B_{S}^{k}=T$. When $S=\{i\}$, we omit the bracket in $B_{\{i\}}^{k}$, i.e., $B_{i}^{k}$ is the union at level $k$ that includes player $i$.

We call the elements in a partition $B$ of $N$ that constitute a coalition by acting as bargaining units the direct members of this coalition, i.e., for any $S=\bigcup_{T \in A} T$ for some $A \subseteq B, N_{B}(S)=\{T: T \subseteq S, T \in B\}$ is the set of direct members of $S$ with respect to $B$. For simplification, we write the set of direct members of $S \in B^{k+1}$ with respect to the $k$ th level of $\mathcal{B}$ as $N_{k}(S)$.

We call $\mathcal{B}$ on $N$ a trivial levels structure, denoted by $\bar{N}$, if $h=1$.

A triple $(N, v, \mathcal{B})$, where $(N, v) \in G^{N}$ and $\mathcal{B}$ is a levels structure on $N$, represents a cooperative game with levels structure, which describes the following cooperative situation. The players first organize themselves into a coalition structure $B^{1}$ (the first level of $\mathcal{B}$ ) as pressure groups for the division of $v(N)$; then the coalitions in $B^{1}$ as players organize themselves again into the coalition structure $B^{2}$ (the second level of $\mathcal{B}$ ); and so until the final level of $\mathcal{B}$ is reached. The set of cooperative games with levels structures on $N$ is denoted by $L G^{N}$. 


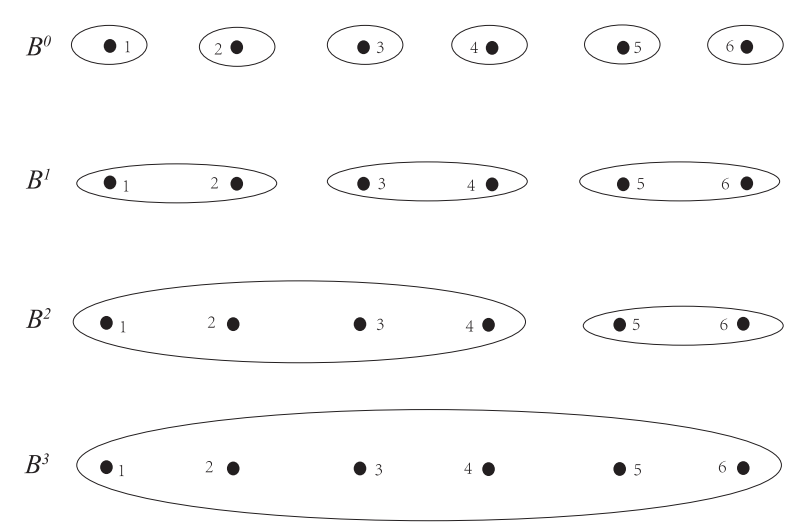

Figure 1. $\mathcal{B}$ in Example 2.2.

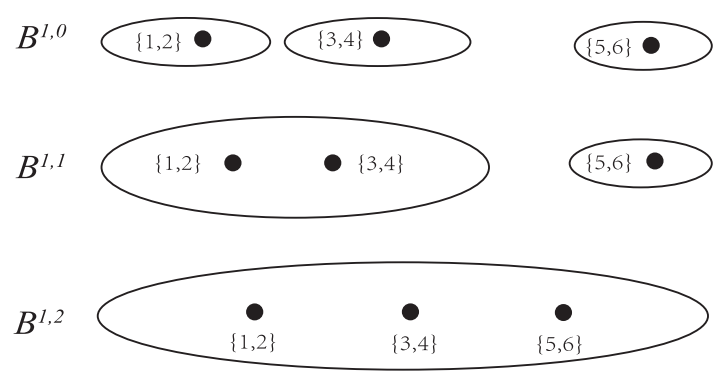

Figure 2. $\mathcal{B} / B^{1}$ in Example 2.2 .

A solution on $N$ for cooperative games with levels structures is a real-valued function $\Psi: L G^{N} \rightarrow \mathbb{R}^{n}$ which assigns to any cooperative game with levels structure an $n$-dimensional payoff vector.

For each $k \in\{0,1, \ldots, h\}$, we define a game with levels structure $\left(B^{k}, v / B^{k}, \mathcal{B} / B^{k}\right)$ on $B^{k}$, induced from $(N, v, \mathcal{B})$ by viewing unions at level $k$ as individual players. Indeed, $B^{k}$ is the set of players at level $k$ in the $k$-level game $\left(B^{k}, v / B^{k}\right)$. The worth of a coalition of players at level $k$ in the game $\left(B^{k}, v / B^{k}\right)$ is defined in a natural way, as the worth of the subset of all original players that it contains. Formally, the worth of the subset $\left\{S_{1}, S_{2}, \ldots, S_{t}\right\} \subseteq B^{k}$ of players at level $k$ is defined to be $\left(v / B^{k}\right)\left(\left\{S_{1}, S_{2}, \ldots, S_{t}\right\}\right)=v\left(S_{1} \cup S_{2} \ldots \cup S_{t}\right)$. Of course, $\mathcal{B} / B^{k}=\left(B^{k, 0}, B^{k, 1}, \ldots, B^{k, h-k}\right)$ is again a levels structure of degree $h-k$ starting with the $k$ th level of $\mathcal{B}$ given by: for all $r \in\{0,1, \ldots, h-k\}, B^{k, r}=\left\{\left\{U: U \in B^{k}, U \subseteq U^{\prime}\right\}: U^{\prime} \in B^{k+r}\right\}$. In order to try to simplify the notations, we write $\left(B^{k}, v / B^{k}\right)$ as $\left(B^{k}, v\right)$, and $\left(B^{k}, v / B^{k}, \mathcal{B} / B^{k}\right)$ as $\left(B^{k}, v, \mathcal{B}\right)$, if there is no confusion.

Example 2.2. Let $\mathcal{B}=\left(B^{0}, B^{1}, B^{2}, B^{3}\right)$ be a levels structure of degree 3 given by $B^{0}=\{\{1\},\{2\},\{3\}$, $\{4\},\{5\},\{6\}\}, B^{1}=\{\{1,2\},\{3,4\},\{5,6\}\}, B^{2}=\{\{1,2,3,4\},\{5,6\}\}$, and $B^{3}=\{\{1,2,3,4,5,6\}\}$ showed in Figure 1.

The restriction of $\mathcal{B}$ to the subset $\{1,2,3,4\}$ is a levels structure of degree 2, i.e., $\mathcal{B}(\{1,2,3,4\})=$ $(\{\{1\},\{2\},\{3\},\{4\}\},\{\{1,2\},\{3,4\}\},\{\{1,2,3,4\}\})$.

The levels structure $\mathcal{B}$ by viewing the unions at the first level as individuals is $\mathcal{B} / B^{1}=$ $\left(B^{1,0}, B^{1,1}, B^{1,2}\right)$ where $B^{1,0}=\{\{\{1,2\}\},\{\{3,4\}\},\{\{5,6\}\}\}, B^{1,1}=\{\{\{1,2\},\{3,4\}\},\{\{5,6\}\}\}$, and $B^{1,2}=$ $\{\{\{1,2\},\{3,4\},\{5,6\}\}\}$, as depicted in Figure 2 . 


\section{The Multi-Step Shapley Value and its axiomatizations}

In this section we extend the two-step Shapley value [7] to cooperative games with levels structures and define the multi-step Shapley value. Without special instructions, the levels structure $\mathcal{B}$ we mentiond in the following context has the degree $h$, denoted as $\mathcal{B}=\left(B^{0}, B^{1}, \ldots, B^{h}\right)$.

Definition 3.1. The multi-step Shapley value $M S(N, v, \mathcal{B})$ for all $(N, v, \mathcal{B}) \in L G^{N}$ is defined by

$$
M S_{i}(N, v, \mathcal{B})=S h_{i}\left(B_{i}^{1}, v\right)+\sum_{k=2}^{h} \frac{S h_{B_{i}^{k-1}}\left(N_{k-1}\left(B_{i}^{k}\right), v\right)-v\left(B_{i}^{k-1}\right)}{\prod_{l=1}^{k-1}\left|N_{l-1}\left(B_{i}^{l}\right)\right|}
$$

for all $i \in N$, where $\left(N_{k-1}\left(B_{i}^{k}\right), v\right)$ is the restriction to the subset of players $N_{k-1}\left(B_{i}^{k}\right)$ of the $k-1$ level game.

We can interpret the multi-step Shapley value by the following multi-step bargaining process.

In the first step, all the players forming the union $S^{1} \in B^{1}$ at the first level bargain for the worth of $S^{1}$ and agree that $v\left(S^{1}\right)$ is distributed by the Shapley value of the game restricted to the subset $S_{1}$. So, each player $i \in S^{1}$ receives $S h_{i}\left(S^{1}, v\right)$ in this step.

In the step $\mathrm{k}(k \in\{2,3, \ldots, h-1\})$, all the unions at level $k-1$ act like single players. They agree that the worth of each formed union $S^{k} \in B^{k}$ at level $k$ is distributed among its direct members by the Shapley value, i.e., each member $S^{k-1} \in N_{k-1}\left(S^{k}\right)$ receives $S h_{S^{k-1}}\left(N_{k-1}\left(S^{k}\right), v\right)$. However, players in $S^{k-1}$ have already got a total of $v\left(S^{k-1}\right)$ in the previous $k-1$ steps. So, the generated surplus $S h_{S^{k-1}}\left(N_{k-1}\left(S^{k}\right), v\right)-v\left(S^{k-1}\right)$ is allocated among all the original players in $S^{k-1}$ in a way that each union at a certain level is treated equally, i.e., each player gets the proportion $\frac{1}{\prod_{l=1}^{k-1}\left|N_{l-1}\left(B_{i}^{l}\right)\right|}$ of the surplus.

Finally, the sum of the payments received by each player over all steps is its multi-step Shapley value.

Remark 3.2. In particular, when the degree of the levels structure $\mathcal{B}$ on $N$ is 2, i.e., $\mathcal{B}=\left(B^{0}, B^{1}, B^{2}\right), \mathcal{B}$ corresponds to the coalition structure $B^{1}$. The difference is that in the model of coalition structure [11], $B^{1}$ is a partition that divides the player set $N$ into disjoint groups, which can cooperate, but in the levels structure, the cooperation between these disjoint groups needs to be described by the last level $B^{2}=\{N\}$. Hence, the multistep Shapley value for $(N, v, \mathcal{B})$ where $\mathcal{B}=\left(B^{0}, B^{1}, B^{2}\right)$, coincides with the two-step Shapley value of the game $\left(N, v, B^{1}\right)$ with the coalition structure $B^{1}$, i.e., for any $i \in N, M S_{i}(N, v, \mathcal{B})=S h_{i}\left(B_{i}^{1}, v\right)+\frac{S h_{B_{i}^{1}}\left(B^{1}, v\right)-v\left(B_{i}^{1}\right)}{\left|B_{i}^{1}\right|}=$ $T S_{i}\left(N, v, B^{1}\right)$.

In the following proposition, we state that the sum of the multi-step Shapley values of players in a union at a level is the payoff of this union when we treat it as a unit.

Proposition 3.3. For any $S \in B^{k}, k \in\{1,2, \ldots, h-1\}$,

$$
\sum_{i \in S} M S_{i}(N, v, \mathcal{B})=M S_{S}\left(B^{k}, v, \mathcal{B}\right)
$$

holds.

Proof. Let $S \in B^{k}, k \in\{1,2, \ldots, h-1\}$. According to Definition 3.1, on one hand, we have 


$$
\begin{aligned}
\sum_{i \in S} M S_{i}(N, v, \mathcal{B})= & \sum_{T_{1} \in N_{k-1}(S)} \sum_{T_{2} \in N_{k-2}\left(T_{1}\right)} \ldots \sum_{T_{k-1} \in N_{1}\left(T_{k-2}\right)} \sum_{i \in T_{k-1}} M S_{i}(N, v, \mathcal{B}) \\
= & \sum_{T_{1} \in N_{k-1}(S)} \ldots \sum_{T_{2} \in N_{k-2}\left(T_{1}\right)} \ldots \sum_{T_{k-1} \in N_{1}\left(T_{k-2}\right)}\left[S h_{T_{k-1}}\left(N_{1}\left(T_{k-2}\right), v\right)\right. \\
& \left.+\sum_{m=3}^{h} \frac{S h_{B_{T_{k-2}-1}^{m-1}}\left(N_{m-1}\left(B_{T_{k-2}}^{m}\right), v\right)-v\left(B_{T_{k-2}}^{m-1}\right)}{\prod_{l=2}^{m-1}\left|N_{l-1}\left(B_{T_{k-2}}^{l}\right)\right|}\right] \\
= & \sum_{T_{1} \in N_{k-1}(S)}\left[S h_{T_{1}}\left(N_{k-1}(S), v\right)+\sum_{m=k+1}^{h} \frac{S h_{B_{S}^{m-1}}\left(N_{m-1}\left(B_{S}^{k}\right), v\right)-v\left(B_{S}^{m-1}\right)}{\prod_{l=k}^{m-1}\left|N_{l-1}\left(B_{S}^{l}\right)\right|}\right] \\
= & S h_{S}\left(N_{k}\left(B_{S}^{k+1}\right), v\right)+\sum_{m=k+2}^{h} \frac{S h_{B_{S}^{m-1}}\left(N_{m-1}\left(B_{S}^{m}\right), v\right)-v\left(B_{S}^{m-1}\right)}{\prod_{l=k+1}^{m-1}\left|N_{l-1}\left(B_{S}^{l}\right)\right|} \\
= & S h_{S}\left(N_{k}\left(B_{S}^{k+1}\right), v\right)+\sum_{m=k+1}^{h-1} \frac{S h_{B_{S}^{m}}\left(N_{m}\left(B_{S}^{m+1}\right), v\right)-v\left(B_{S}^{m}\right)}{\prod_{l=k+1}^{m}\left|N_{l-1}\left(B_{S}^{l}\right)\right|} .
\end{aligned}
$$

On the other hand,

$$
\begin{aligned}
M S_{S}\left(B^{k}, v, \mathcal{B}\right) & =S h_{S}\left(N_{k}\left(B_{S}^{k+1}\right), v\right)+\sum_{m=2}^{h-k} \frac{S h_{B_{S}^{k+m-1}}\left(N_{k+m-1}\left(B_{S}^{m+k}\right), v\right)-v\left(B_{S}^{k+m-1}\right)}{\prod_{l=1}^{m-1}\left|N_{k+l-1}\left(B_{S}^{k+l}\right)\right|} \\
& =S h_{S}\left(N_{k}\left(B_{S}^{k+1}\right), v\right)+\sum_{m=k+1}^{h-1} \frac{S h_{B_{S}^{m}}\left(N_{m}\left(B_{S}^{m+1}\right), v\right)-v\left(B_{S}^{m}\right)}{\prod_{l=k+1}^{m}\left|N_{l-1}\left(B_{S}^{l}\right)\right|},
\end{aligned}
$$

where the first equality holds due to Definition 3.1.

Therefore, $\sum_{i \in S} M S_{i}(N, v, \mathcal{B})=M S_{S}\left(B^{k}, v, \mathcal{B}\right)$.

Now we present several properties for a solution $\Psi$ on $L G^{N}$ as below.

Efficiency. $\sum_{i \in N} \Psi_{i}(N, v, \mathcal{B})=v(N)$ for all $(N, v, \mathcal{B}) \in L G^{N}$.

Additivity. $\Psi(N, v+w, \mathcal{B})=\Psi(N, v, \mathcal{B})+\Psi(N, w, \mathcal{B})$ holds for all $(N, v, \mathcal{B}),(N, w, \mathcal{B}) \in L G^{N}$.

Level balanced contributions. Let $k \in\{1,2, \ldots, h\}$ and $S \in B^{k}$. For any $T_{1}, T_{2} \in N_{k-1}(S)$,

$$
\begin{aligned}
& \Psi_{T_{1}}\left(N_{k-1}(S), v, \bar{B}\right)-\Psi_{T_{1}}\left(N_{k-1}(S) \backslash\left\{T_{2}\right\}, v / B^{k-1}, \overline{N_{k-1}(S)} \backslash\left\{T_{2}\right\}\right) \\
& \quad=\Psi_{T_{2}}\left(N_{k-1}(S), v, \bar{B}\right)-\Psi_{T_{2}}\left(N_{k-1}(S) \backslash\left\{T_{1}\right\}, v / B^{k-1}, \overline{N_{k-1}(S)} \backslash\left\{T_{1}\right\}\right),
\end{aligned}
$$

where $\left(N_{k-1}(S), v / B^{k-1}\right)$ is the restriction to the subset $N_{k-1}(S)$ of players in the game of level $k-1$, $\overline{N_{k-1}(S)}$ is the trivial levels structure on the set $N_{k-1}(S)$ and $\overline{N_{k-1}(S)} \backslash\left\{T_{1}\right\}\left(\overline{N_{k-1}(S)} \backslash\left\{T_{2}\right\}\right)$ is the trivial levels structure on the set $N_{k-1}(S)$ after deleting $T_{1}\left(T_{2}\right)$ for all $(N, v, \mathcal{B}) \in L G^{N}$. In the following context, we will write $\left(N_{k-1}(S), v / B^{k-1}\right)$ as $\left(N_{k-1}(S), v\right),\left(N_{k-1}(S), v / B^{k-1}, \overline{N_{k-1}(S)}\right)$ as $\left(N_{k-1}(S), v, \bar{B}\right)$, and $\left(N_{k-1}(S) \backslash\{T\}, v / B^{k-1}, \overline{N_{k-1}(S)} \backslash\{T\}\right)$ as $\left(N_{k-1}(S) \backslash\{T\}, v, \bar{B}\right)$ for any $T \in N_{k-1}(S)$, if there is no confusion. 
This property is a generalization of the coalitional balanced contributions defined by Calvo and Gutiérrez [2]. It says that for each union at a level, the amount that a direct member would obtain or lose from the withdrawal of another direct member is equal.

Internal surplus equality. Let $k \in\{1,2, \ldots, h\}$ and $S \in B^{k}$. For any $S_{1}, S_{2} \in N_{k-1}(S)$,

$$
\sum_{i \in S_{1}} \Psi_{i}(N, v, \mathcal{B})-\Psi_{S_{1}}\left(N_{k-1}(S), v, \bar{B}\right)=\sum_{i \in S_{2}} \Psi_{i}(N, v, \mathcal{B})-\Psi_{S_{2}}\left(N_{k-1}(S), v, \bar{B}\right),
$$

for all $(N, v, \mathcal{B}) \in L G^{N}$.

This property states that the direct members of a union at a level must have an equal share of the surplus earned by the union as a unit.

Level strong monotonicity in unions. Let $k \in\{1, \ldots, h\}$ and $T$ be a direct member of $S \in B^{k}$ with respect to $B^{k-1}$, i.e., $T \in N_{k-1}(S)$. If for all $A \subseteq N_{k-1}(S) \backslash\{T\},\left(v / B^{k-1}\right)(A \cup\{T\})-\left(v / B^{k-1}\right)(A) \geq\left(w / B^{k-1}\right)(A \cup$ $\{T\})-\left(w / B^{k-1}\right)(A)$, then

$$
\Psi_{T}\left(N_{k-1}(S), v, \bar{B}\right) \geq \Psi_{T}\left(N_{k-1}(S), w, \bar{B}\right)
$$

for all $(N, v, \mathcal{B}),(N, w, \mathcal{B}) \in L G^{N}$.

This property states that when the direct players of a union at some level bargain for its worth, the division of the direct member whose marginal contribution increases should not be decreased.

Internal symmetry in unions. Let $k \in\{1,2, \ldots, h\}$ and $S \in B^{k}$. For any $S_{1}, S_{2} \in N_{k-1}(S)$, if for all $A \subseteq N_{k-1}(S) \backslash\left\{S_{1}, S_{2}\right\},\left(v / B^{k-1}\right)\left(A \cup\left\{S_{1}\right\}\right)-\left(v / B^{k-1}\right)(A)=\left(v / B^{k-1}\right)\left(A \cup\left\{S_{2}\right\}\right)-\left(v / B^{k-1}\right)(A)$ holds, then

$$
\sum_{i \in S_{1}} \Psi_{i}(N, v, \mathcal{B})=\sum_{i \in S_{2}} \Psi_{i}(N, v, \mathcal{B})
$$

for all $(N, v, \mathcal{B}) \in L G^{N}$.

This property says that the symmetric direct members of a union at any level receive equal payoffs.

Level null coalition property. Let $k \in\{1,2, \ldots, h\}$ and $S \in B^{k}$. For some $T \in N_{k-1}(S)$, if for all $A \subseteq$ $N_{k-1}(S) \backslash\{T\},\left(v / B^{k-1}\right)(A \cup\{T\})-\left(v / B^{k-1}\right)(A)=0$, then

$$
\Psi_{T}\left(N_{k-1}(S), v, \bar{B}\right)=0
$$

for all $(N, v, \mathcal{B}) \in L G^{N}$.

This property claims that the direct member of a union at a level, who has no contribution to the worth of this union, receives zero from this union.

The theorem below gives a characterization of the multi-step Shapley value for cooperative games with levels structures.

Theorem 3.4. The multi-step Shapley value is the unique allocation rule for cooperative games with levels structures satisfying efficiency, level balanced contributions, and internal surplus equality.

Proof. Let $(N, v, \mathcal{B})$ be a cooperative game with levels structure. We first prove that the multi-step Shapley value $M S(N, v, \mathcal{B})$ satisfies efficiency, level balanced contributions, and internal surplus equality.

Efficiency. According to Definition 3.1 and Proposition 3.3, we have

$$
\begin{aligned}
\sum_{i \in N} M S_{i}(N, v, \mathcal{B}) & =\sum_{S \in B^{h-1}} \sum_{i \in S} M S_{i}(N, v, \mathcal{B}) \\
& =\sum_{S \in B^{h-1}} M S_{S}\left(B^{h-1}, v, \mathcal{B}\right) \\
& =\sum_{S \in B^{h-1}} S h_{S}\left(B^{h-1}, v\right)=v(N) .
\end{aligned}
$$


Level balanced contributions. For any $S \in B^{k}, k \in\{1,2, \ldots, h\}$, by the definition of the multi-step Shapley value, $M S\left(N_{k-1}(S), v, \bar{B}\right)=S h\left(N_{k-1}(S), v\right)$ because $\overline{N_{k-1}(S)}$ is a trivial levels structure. Adding the balanced contributions of the Shapley value [10], we can easily verify the level balanced contributions property of the multi-step Shapley value.

Internal surplus equality. Let $k \in\{1,2, \ldots, h\}$ and $S \in B^{k}$. We have

$$
\sum_{i \in T} M S_{i}(N, v, \mathcal{B})=S h_{T}\left(N_{k-1}(S), v\right)+\sum_{m=k}^{h-1} \frac{S h_{B_{S}^{m}}\left(N_{m}\left(B_{S}^{m+1}\right), v\right)-v\left(B_{S}^{m}\right)}{\prod_{l=k}^{m}\left|N_{l-1}\left(B_{S}^{l}\right)\right|},
$$

for any $T \in N_{k-1}(S)$ by the proof of Proposition 3.3. Therefore, for any $S_{1}, S_{2} \in N_{k-1}(S)$,

$$
\begin{aligned}
\sum_{i \in S_{1}} M S_{i}(N, v, \mathcal{B})-\sum_{i \in S_{2}} M S_{i}(N, v, \mathcal{B}) & =S h_{S_{1}}\left(N_{k-1}(S), v\right)-S h_{S_{2}}\left(N_{k-1}(S), v\right) \\
& =M S_{S_{1}}\left(N_{k-1}(S), v, \bar{B}\right)-M S_{S_{2}}\left(N_{k-1}(S), v, \bar{B}\right),
\end{aligned}
$$

where the first equality holds because of Equation (3.1) and the second equality holds by Definition 3.1.

Next, we prove the uniqueness of the allocation rule $\Psi$ satisfying the efficiency, level balanced contributions, and internal surplus equality. Define $\left|N_{k-1}(S)\right|=l$, we first prove that $\Psi\left(N_{k-1}(S), v, \bar{B}\right)=S h\left(N_{k-1}(S), v\right)$ by induction on $l$.

If $l=1$, we can easily verify that $\Psi\left(N_{k-1}(S), v, \bar{B}\right)=S h\left(N_{k-1}(S), v, \bar{B}\right)$. Assume that when $\left|N_{k-1}(S)\right|=l$, $\Psi\left(N_{k-1}(S), v, \bar{B}\right)=S h\left(N_{k-1}(S), v, \bar{B}\right)$.

Suppose $\left|N_{k-1}(S)\right|=l+1$. It follows from level balanced contributions that

$$
\Psi_{T_{1}}\left(N_{k-1}(S), v, \bar{B}\right)-\Psi_{T_{2}}\left(N_{k-1}(S), v, \bar{B}\right)=\Psi_{T_{1}}\left(N_{k-1}(S) \backslash\left\{T_{2}\right\}, v, \bar{B}\right)-\Psi_{T_{2}}\left(N_{k-1}(S) \backslash\left\{T_{1}\right\}, v, \bar{B}\right) .
$$

Using this equation and efficiency iteratively, we obtain that $\Psi\left(N_{k-1}(S), v, \bar{B}\right)$ is unique and equal to $S h\left(N_{k-1}(S), v, \bar{B}\right)$. Further, in the case of $k=h$, using internal surplus equality and efficiency iteratively, we have that $\sum_{i \in S} \Psi_{i}(N, v, \mathcal{B})$ for each $S \in B^{h-1}$ is unique. Continue this operation from $k=h$ to $k=1$, we can finally prove that $\Psi(N, v, \mathcal{B})$ is unique.

Therefore, the multi-step Shapley value is the unique allocation rule for cooperative games with levels structures satisfying efficiency, level balanced contributions, and internal surplus equality.

By replacing level balanced contributions with level strong monotonicity in unions and internal symmetry in unions in Theorem 3.4, we derive another characterization of the multi-step Shapley value.

Theorem 3.5. The multi-step Shapley value is the unique allocation rule for cooperative games with levels structures satisfying efficiency, internal surplus equality, level strong monotonicity in unions and internal symmetry in unions.

Proof. Let $(N, v, \mathcal{B})$ be a cooperative game with levels structure. We first prove that the multi-step Shapley value $M S(N, v, \mathcal{B})$ satisfies the properties mentioned in this theorem. Efficiency and internal surplus equality of $M S(N, v, \mathcal{B})$ have been proven in Theorem 3.4. Level strong monotonicity in unions of the multi-step Shapley value is obvious due to for any $S \in B^{k}, k \in\{1, \ldots, h\}, M S\left(N_{k-1}(S), v, \bar{B}\right)=S h\left(N_{k-1}(S), v\right)$.

Internal symmetry in unions. Let $k \in\{1,2, \ldots, h\}, S \in B^{k}$ and $S_{1}, S_{2} \in N_{k-1}(S)$. On one hand, because of symmetry of the Shapley value [13], if for all $A \subseteq N_{k-1}(S) \backslash\left\{S_{1}, S_{2}\right\},\left(v / B^{k-1}\right)\left(A \cup\left\{S_{1}\right\}\right)-\left(v / B^{k-1}\right)(A)=$ $\left(v / B^{k-1}\right)\left(A \cup\left\{S_{2}\right\}\right)-\left(v / B^{k-1}\right)(A)$, then

$$
S h_{S_{1}}\left(N_{k-1}(S), v\right)-S h_{S_{2}}\left(N_{k-1}(S), v\right)=0 .
$$


On the other hand, by Proposition 3.3,

$$
\begin{aligned}
& \sum_{i \in S_{1}} M S_{i}(N, v, \mathcal{B})=S h_{S_{1}}\left(N_{k-1}(S), v\right)+\sum_{m=k}^{h-1} \frac{S h_{B_{S}^{m}}\left(N_{m}\left(B_{S}^{m+1}\right), v\right)-v\left(B_{S}^{m}\right)}{\prod_{l=k}^{m}\left|N_{l-1}\left(B_{S}^{l}\right)\right|}, \\
& \sum_{i \in S_{2}} M S_{i}(N, v, \mathcal{B})=S h_{S_{2}}\left(N_{k-1}(S), v\right)+\sum_{m=k}^{h-1} \frac{S h_{B_{S}^{m}}\left(N_{m}\left(B_{S}^{m+1}\right), v\right)-v\left(B_{S}^{m}\right)}{\prod_{l=k}^{m}\left|N_{l-1}\left(B_{S}^{l}\right)\right|} .
\end{aligned}
$$

Therefore,

$$
\sum_{i \in S_{1}} M S_{i}(N, v, \mathcal{B})-\sum_{i \in S_{2}} M S_{i}(N, v, \mathcal{B})=S h_{S_{1}}\left(N_{k-1}(S), v\right)-S h_{S_{2}}\left(N_{k-1}(S), v\right)=0 .
$$

Next, let $\Psi$ be the allocation rule for $(N, v, \mathcal{B})$ satisfying efficiency, internal surplus equality, level strong monotonicity in unions and internal symmetry in unions.

Now we prove the uniqueness of $\Psi(N, v, \mathcal{B})$, by first showing that for any $S \in B^{k}(k \in\{1,2, \ldots, h\})$, $\Psi\left(N_{k-1}(S), v, \bar{B}\right)=S h\left(N_{k-1}(S), v\right)$. It is known that any game can be expressed by the unanimity games, i.e., $v / B^{k-1}=\sum_{\emptyset \subseteq A \subseteq N_{k-1}(S)} \alpha_{A} u_{A}$, and $S h_{T}\left(N_{k-1}(S), v\right)=\sum_{T \in A, A \subseteq N_{k-1}(S)} \frac{\alpha_{A}}{|A|}$, where for the unanimity game $u_{A}, u_{A}(E)$ is equal to 1 if $A \subseteq E \subseteq N_{k-1}(S)$ and 0 otherwise.

Define the index $I$ of $v / B^{k-1}$ to be the minimal number of non-zero items in the expression $v / B^{k-1}=$ $\sum_{\emptyset \subseteq A \subseteq N_{k-1}(S)} \alpha_{A} u_{A}, \Psi\left(N_{k-1}(S), v, \bar{B}\right)=S h\left(N_{k-1}(S), v\right)$ is proved by induction on $I$.

If $I=0$, then $v / B^{k-1}=0$ and so $\Psi\left(N_{k-1}(S), v, \bar{B}\right)=0=\operatorname{Sh}\left(N_{k-1}(S), v\right)$.

If $I=1$, then $v / B^{k-1}=\alpha_{A} u_{A}$ for some $A \subsetneq N_{k-1}(S)$. Following internal symmetry in unions and efficiency, $\Psi_{T}\left(N_{k-1}(S), v, \bar{B}\right)$ is equal to $\frac{\alpha_{A}}{|A|}$ for any $T \in A$. For any $T \in N_{k-1}(S)$, since $\left(v / B^{k-1}\right)(A \cup\{T\})-\left(v / B^{k-1}\right)(A)=$ 0 for all $A \subseteq N_{k-1}(S) \backslash\{T\}$, it follows from level strong monotonicity that $\Psi_{T}\left(N_{k-1}(S), v, \bar{B}\right)=0$. So $\Psi\left(N_{k-1}(S), v, \bar{B}\right)=S h\left(N_{k-1}(S), v\right)$.

Assume $\Psi\left(N_{k-1}(S), v, \bar{B}\right)=S h\left(N_{k-1}(S), v\right)$ whenever the index of $v / B^{k-1}$ is at most $I$, and let $v / B^{k-1}$ has the index $I+1$ with the expression $v / B^{k-1}=\sum_{k=1}^{I+1} \alpha_{A_{k}} u_{A_{k}}$, all $\alpha_{A_{k}} \neq 0$.

Let $A=\cap_{k=1}^{I+1} A_{k}$ and suppose that $T \notin A$. Define the game $w / B^{k-1}=\sum_{k ; T \in A_{k}} \alpha_{A_{k}} u_{A_{k}}$. The index of $w / B^{k-1}$ is at most $I$ and $\left(v / B^{k-1}\right)(A \cup\{T\})-\left(v / B^{k-1}\right)(A)=\left(w / B^{k-1}\right)(A \cup\{T\})-\left(w / B^{k-1}\right)(A)$ for all $A \subseteq N_{k-1}(S) \backslash\{T\}$. So by level strong monotonicity and induction it follows that $\Psi_{T}\left(N_{k-1}(S), v, \bar{B}\right)=\Psi_{T}\left(N_{k-1}(S), w, \bar{B}\right)=$ $\sum_{k: T \in A_{k}} \frac{\alpha_{A_{k}}}{\left|A_{k}\right|}=S h_{T}\left(N_{k-1}(S), v, \bar{B}\right)$. For $T \in A=\cap_{k=1}^{I+1} A_{k}, \Psi_{T}\left(N_{k-1}(S), v, \bar{B}\right)=S h_{T}\left(N_{k-1}(S), v, \bar{B}\right)$ by efficiency and internal symmetry in unions. Therefore, $\Psi\left(N_{k-1}(S), v, \bar{B}\right)=S h\left(N_{k-1}(S), v, \bar{B}\right)$ is proved.

Further, we can adopt the same method in Theorem 1 using internal surplus equality and efficiency repeatedly to show that $\Psi(N, v, \mathcal{B})$ is unique and equal to $M S(N, v, \mathcal{B})$.

Therefore, the multi-step Shapley value is the unique allocation rule satisfying the efficiency, internal surplus equality, level strong monotonicity in unions and internal symmetry in unions for cooperative games with levels structures.

If additivity and level null coalition property in Theorem 3.5 are substituted by level strong monotonicity in unions, we have the following theorem.

Theorem 3.6. The multi-step Shapley value is the unique allocation rule for cooperative games with levels structures satisfying efficiency, internal surplus equality, internal symmetry in unions, additivity, and level null coalition property. 
Proof. Efficiency, internal surplus equality and internal symmetry in unions of the multi-step Shapley value have been proven in Theorem 3.4. Additivity can be easily observed from Definition 3.1. Level null coalition property is obtained by the fact of $M S\left(N_{k-1}(S), v, \bar{B}\right)=S h\left(N_{k-1}(S), v\right)$ for any $S \in B^{k}, k \in\{1,2, \ldots, h\}$ and null player property (see Shapely [13]) of the Shapley value.

Given an allocation rule $\Psi$ for $(N, v, \mathcal{B})$ that satisfies the properties in this theorem. It is not difficult to prove that for any $S \in B^{k}, k \in\{1,2, \ldots, h\}, \Psi\left(N_{k-1}(S), v, \bar{B}\right)=S h\left(N_{k-1}(S), v\right)$ holds from efficiency, internal symmetry in unions, additivity and level null coalition property by the aid of the axiomatization of the Shapley value in Shapley [13], and we omit the proof. Thus, applying the internal surplus equality for all $S \in B^{k}, k \in$ $\{1,2, \ldots, h\}$, and efficiency, we can derive that $\Psi(N, v, \mathcal{B})$ is equal to the multi-step Shapley value.

Therefore, the multi-step Shapley value is the unique allocation rule that satisfies efficiency, internal surplus equality, internal symmetry in unions, additivity and level null coalition property for cooperative games with levels structures.

\section{A NON-COOPERATIVE Approach The MUlti-Step Shapley VAlue}

In this section, we design a non-cooperative multi-step bidding mechanism that implements the multi-step Shapley value for games with levels structures, inspired by the bidding mechanism of Pérez-Castrillo and Wettstein [12], and the levels bidding mechanism of Vidal-Puga [17].

Our mechanism is played in several rounds and defined recursively. Once we know the rules of multi-step bidding mechanism when the levels structure has at most degree $h$, we know define the mechanism for levels structure with degree $h$. In the first round, the players in each union at the first level simultaneously bargain for the resources of their own coalition. Let $B_{p} \in B^{1}$. Each player $i \in B_{p}$ announces a bid $b_{j}^{i} \in \mathbb{R}$ for any $j \in B_{p} \backslash\{i\}$. The bid $b_{j}^{i}$ can be seen as the amount that player $i$ is willing to pay to player $j$ as the cost of being elected as the proposer. The net bid of a player is the difference between what it gives to others and what it receives from others. The player, say $\alpha_{p}$, in $B_{p}$ who makes the highest net bid will be chosen as the proposer. If several players make the highest net bid, the proposer is chosen randomly among them. The proposer $\alpha_{p}$ pays the promised bids to players in $B_{p} \backslash\{i\}$. Then, the proposer $\alpha_{p}$ makes an offer $y^{\alpha_{p}}$ to the other players in $B_{p}$. If all the players in $B_{p} \backslash\left\{\alpha_{p}\right\}$ accept the offer, the proposer $\alpha_{p}$ becomes a representative of the coalition $B_{p}$, i.e., $\alpha_{p}$ goes to Round 2 with all the resources of $B_{p}$. If at least one player in $B_{p} \backslash\left\{\alpha_{p}\right\}$ rejects the offer, the proposer $\alpha_{p}$ leaves the game and other players in $B_{p} \backslash \alpha_{p}$ continue to play the game $\left(B_{p} \backslash\left\{\alpha_{p}\right\}, v, \overline{B_{p} \backslash\left\{\alpha_{p}\right\}}\right)$ (If there is no confusion, in the following context, we abbreviate $\left(B_{p} \backslash\left\{\alpha_{p}\right\}, v, \overline{B_{p} \backslash\left\{\alpha_{p}\right\}}\right)$ as $\left.\left(B_{p} \backslash\left\{\alpha_{p}\right\}, v, \bar{B}\right)\right)$. When there is only one player in $B_{p}$, the player becomes the representative of itself.

After finishing Round 1 , denote by $R$ the set of the remaining players after removing all the players in the unions at the first level that do not have their own representatives.

Let $U$ be a maximal coalition that can be formed by the players in $R$, i.e., $U \in B^{k}$ for some $k \in\{1,2, \ldots, h\}$ is a subset of $R$ and there exists no $U^{\prime} \in B^{m}$ for some $m \in\{1,2, \ldots, h\}$ such that $U \subsetneq U^{\prime}$, since the formation of a coalition requires the participation of all its members.

In the second round, all the coalitions in $N_{1}(U)$ act as individual players and play $\left(B^{1}(U), v / B^{1}, \mathcal{B}(U) / B^{1}(U)\right)$ following the same procedure as before. Proceed this until in some round, there is only one player who plays alone and obtains its individual worth.

Now we formally describe the multi-step bidding mechanism and proceed by the induction on the degree $h$ of levels structure $\mathcal{B}$ as follows.

If there is only one player, then this player obtains the worth of its stand-alone coalition.

If $h=1$, the players play a single round. This round comprises the bidding mechanism (Pérez-Castrillo and Wettstein [12]) associated with the game $(N, v)$.

Suppose that we know the multi-step bidding mechanism when the degree of $\mathcal{B}$ is less than $h(h>1)$. For the levels structure with degree $h$, the multi-step bidding mechanism proceeds as follows:

Round 1. Let $B_{p} \in B^{1}$. 
Stage 1. Each player $i \in B_{p}$ makes bids $b_{j}^{i} \in \mathbb{R}$ for each $j \in B_{p} \backslash\{i\}$. Hence, at this stage, a strategy for player $i \in B_{p}$ is a vector $b^{i} \in \mathbb{R}^{\left|B_{p}\right|-1}$. Calculate the net bid

$$
B_{i}=\sum_{j \in B_{p} \backslash\{i\}} b_{j}^{i}-\sum_{j \in B_{p} \backslash\{i\}} b_{i}^{j}
$$

for each $i \in B_{p}$. Let $\alpha_{p}$ be the player with the highest net bid among players in $B_{p}$ ( $\alpha_{p}$ is randomly chosen in case of a non-unique maximizer). Then $\alpha_{p}$ becomes the proposer in the next stage.

Stage 2. The proposer $\alpha_{p}$ makes an offer $y_{i}^{\alpha_{p}}$ to each player $i \in B_{p} \backslash\left\{\alpha_{p}\right\}$. Therefore, at this stage a strategy for $\alpha_{p}$ is a vector $y^{\alpha_{p}} \in \mathbb{R}^{\left|B_{p}\right|-1}$.

Stage 3. The players of $B_{p} \backslash\left\{\alpha_{p}\right\}$ either accept or reject the offer. If at least one player rejects it, then we call the offer rejected. Otherwise, we call the offer accepted.

If the offer is rejected, player $\alpha_{p}$ pays $b_{i}^{\alpha_{p}}$ to each $i \in B_{p} \backslash\left\{\alpha_{p}\right\}$ and leaves the non-cooperative game with the payoff $v\left(\left\{\alpha_{p}\right\}\right)-\sum_{i \in B_{p} \backslash\left\{\alpha_{p}\right\}} b_{i}^{\alpha_{p}}$. The other players in $B_{p} \backslash\left\{\alpha_{p}\right\}$ play the multi-step bidding mechanism $\left(B_{p} \backslash\left\{\alpha_{p}\right\}, v, \bar{B}\right)$. Any player $i \in B_{p} \backslash\left\{\alpha_{p}\right\}$ obtains as its final payoff the sum of the received bid $b_{i}^{\alpha_{p}}$ and the outcome of the multi-step bidding mechanism corresponding to $\left(B_{p} \backslash\left\{\alpha_{p}\right\}, v, \bar{B}\right)$.

If the offer is accepted, $\alpha_{p}$ receives $P_{\alpha_{p}}^{1}=-\sum_{i \in B_{p} \backslash\left\{\alpha_{p}\right\}}\left(b_{i}^{\alpha_{p}}+y_{i}^{\alpha_{p}}\right)$ in this round and becomes a representative of $B_{p}$. That is, player $\alpha_{p}$ goes to Round 2 with all resources of $B_{p}$. Each $i \in B_{p} \backslash\left\{\alpha_{p}\right\}$ leaves the non-cooperative game with a final payoff $b_{i}^{\alpha_{p}}+y_{i}^{\alpha_{p}}$.

After finishing Round 1, let $R^{1}$ be the set of remaining players after removing the players of unions at the first level which do not have their own representatives and $U^{*}$ be a maximal coalition that can be formed by players in $R^{1}$.

Round 2 through $\mathbf{h}$. The representatives involved in the coalition $U^{*}$ play the multi-step bidding mechanism associated with

$$
\left(B^{1}\left(U^{*}\right), v / B^{1}\left(U^{*}\right), \mathcal{B}\left(U^{*}\right) / B^{1}\left(U^{*}\right)\right),
$$

where the representative $\alpha_{p}$ of $B_{p} \in B^{1}\left(U^{*}\right)$ takes the role of $B_{p}$. These rounds are well defined by induction on $h$. Denote by $p_{\alpha_{p}}^{2}, \ldots, p_{\alpha_{p}}^{h}$, the outcome of the representative $\alpha_{p}$ in these rounds.

The final payoff of the proposer $\alpha_{p}$ is the sum of its obtained payoffs over all rounds, i.e., $p_{\alpha_{p}}^{1}+p_{\alpha_{p}}^{2}+p_{\alpha_{p}}^{h}$.

In order to characterize the $S P N E$ outcomes of the multi-step bidding mechanism, the following result will be helpful.

Proposition 4.1. Let $(N, v, \mathcal{B})$ be a cooperative game with levels structure where $(N, v)$ is superadditive. For any $B_{p} \in B^{1}$,

$$
v(\{j\})-\sum_{i \in B_{p} \backslash\{j\}}\left[M S_{i}(N, v, \mathcal{B})-M S_{i}\left(B_{p} \backslash\{j\}, v, \bar{B}\right)\right] \leq M S_{j}(N, v, \mathcal{B}),
$$

for all $j \in B_{p}$.

Proof. By efficiency of the multi-step Shapely value, we have

$$
\sum_{i \in B_{p} \backslash\{j\}} M S_{i}\left(B_{p} \backslash\{j\}, v, \bar{B}\right)=v\left(B_{p} \backslash\{j\}\right) .
$$

Then we only need to prove that $\sum_{i \in B_{p}} M S_{i}(N, v, \mathcal{B})-v\left(B_{p} \backslash\{j\}\right) \geq v(\{j\})$.

Since superadditivity of $(N, v)$ implies the superadditivity of the game $\left(N_{k-1}(S), v\right)$ for any $S \in B^{k}, k \in$ $\{1,2, \ldots, h\}$, and the Shapley value of a superadditive game satisfies individual rationality, we have that for any 
$k \in\{1,2, \ldots, h\}, S h_{B_{i}^{k-1}}\left(N_{k-1}\left(B_{i}^{k}\right), v\right) \geq v\left(B_{i}^{k-1}\right)$. Furthermore,

$$
\sum_{i \in B_{p}} M S_{i}(N, v, \mathcal{B})=\sum_{i \in B_{p}}\left[S h_{i}\left(B_{p}, v\right)+\sum_{k=2}^{h} \frac{S h_{B_{i}^{k-1}}\left(N_{k-1}\left(B_{i}^{k}\right), v\right)-v\left(B_{i}^{k-1}\right)}{\prod_{l=1}^{k-1}\left|N_{l-1}\left(B_{i}^{l}\right)\right|}\right] \geq v\left(B_{p}\right) .
$$

Applying superadditivity of $(N, v)$, we obtain that

$$
\sum_{i \in B_{p}} M S_{i}(N, v, \mathcal{B})-v\left(B_{p} \backslash\{j\}\right) \geq v\left(B_{p}\right)-v\left(B_{p} \backslash\{j\}\right) \geq v(\{j\}) .
$$

In order to implement the multi-step Shapley value, throughout the rest of this section, inspired by Moldovanu and Winter [9] and Hart and Mas-Colell [3], we give the following two assumptions.

(a) Each player prefers to be a member of a larger coalition.

(b) A player will choose to accept if he is indifferent to accepting or rejecting a offer.

Theorem 4.2. The multi-step bidding mechanism implements the multi-step Shapley value in every SPNE for superadditive games.

Proof. The proof relies on induction on the degree of the levels structure. When $h=1$, the multi-step Shapley value is equal to the Shapley value and the multi-step bidding mechanism is reduced to the bidding mechanism of Pérez-Castrillo and Wettstein [12]. Thus, the multi-step bidding mechanism implements the multi-step Shapley value in $S P N E$ by the fact that the bidding mechanism implements the Shapley value for zero-monotonic games by Theorem 1 in Pérez-Castrillo and Wettstein [12].

Assume the result is true when the levels structure has the degree $h-1(h>1)$ or less, we now prove that the result is still true when the degree is $h$. First, we prove that the multi-step Shapley value is indeed an $S P N E$ outcome. We explicitly construct an $S P N E$ that yields the multi-step Shapley value. Consider the following strategies:

Round 1. Let $B_{p} \in B^{1}$.

Stage 1. Each player $i \in B_{p}$ announces bids

$$
b_{j}^{i}=M S_{j}(N, v, \mathcal{B})-M S_{j}\left(B_{p} \backslash\{i\}, v, \bar{B}\right)
$$

for every $j \in B_{p} \backslash\{i\}$.

Stage 2. A proposer, player $\alpha_{p}$, offers

$$
y_{j}^{\alpha_{p}}=M S_{j}\left(B_{p} \backslash\{i\}, v, \bar{B}\right)
$$

to every $j \in B_{p} \backslash\left\{\alpha_{p}\right\}$.

Stage 3. Each player $j \in B_{p} \backslash\left\{\alpha_{p}\right\}$ accepts any offer not less than

$$
M S_{j}\left(B_{p} \backslash\{i\}, v, \bar{B}\right)
$$

and rejects any offer strictly less than

$$
M S_{j}\left(B_{p} \backslash\{i\}, v, \bar{B}\right) .
$$

Round 2 through $\mathbf{h}$. The proposers $\alpha_{p}$ in all $B_{p} \in B^{1}$ as representatives play the game $\left(B^{1}, v, \mathcal{B}\right)$ according to strategies of some $S P N E$ with the associated payoff $M S\left(B^{1}, v, \mathcal{B}\right)$. We can find such an $S P N E$ by the induction hypothesis on $h$. 
Now, we prove that these strategies yield the multi-step Shapley value. Note that the offers of $\alpha_{p}$ for any $B_{p} \in B^{1}$ in Round 1 are accepted by players in $B_{p} \backslash\left\{\alpha_{p}\right\}$, then the payoff of $i \in B_{p} \backslash\left\{\alpha_{p}\right\}$ is

$$
\begin{aligned}
b_{i}^{\alpha_{p}}+y_{i}^{\alpha_{p}} & =M S_{i}(N, v, \mathcal{B})-M S_{i}\left(B_{p} \backslash\left\{\alpha_{p}\right\}, v, \bar{B}\right)+M S_{i}\left(B_{p} \backslash\left\{\alpha_{p}\right\}, v, \bar{B}\right) \\
& =M S_{i}(N, v, \mathcal{B}) .
\end{aligned}
$$

The final payoff of proposer $\alpha_{p}$ is

$$
\begin{aligned}
-\sum_{i \in B_{p} \backslash\left\{\alpha_{p}\right\}}\left(b_{i}^{\alpha_{p}}+y_{i}^{\alpha_{p}}\right)+M S_{B_{p}}\left(B^{1}, v, \mathcal{B}\right) & =-\sum_{i \in B_{p} \backslash\left\{\alpha_{q}\right\}} M S_{i}(N, v, \mathcal{B})+\sum_{i \in B_{p}} M S_{i}(N, v, \mathcal{B}) \\
& =M S_{\alpha_{p}}(N, v, \mathcal{B})
\end{aligned}
$$

by Proposition 3.3.

Next, we prove that these strategies are an $S P N E$. It has been easily known that these strategies yields an $S P N E$ by induction on $h$ after Round 2. Therefore, we only need to prove that these strategies induce an $S P N E$ in Round 1. Let $B_{p} \in B^{1}$.

Stage 3. Assume that some player $i \in B_{p} \backslash\left\{\alpha_{p}\right\}$ rejects the offer of the proposer $\alpha_{p}$. The game $\left(B_{p} \backslash\left\{\alpha_{p}\right\}, v, \bar{B}\right)$ is played and the outcome is

$$
M S\left(B_{p} \backslash\left\{\alpha_{p}\right\}, v, \bar{B}\right)
$$

by the induction hypothesis on $h$ because the degree of $\overline{B_{p} \backslash\left\{\alpha_{p}\right\}}$ is less than $h$. Hence, it is optimal for player $i$ to accept any offer not less than

$$
M S_{i}\left(B_{p} \backslash \alpha_{p}, v, \bar{B}\right)
$$

Stage 2. If $\alpha_{p}$ makes an offer to some $i \in B_{p} \backslash\left\{\alpha_{p}\right\}$ less than $M S_{i}\left(B_{p} \backslash\left\{\alpha_{p}\right\}, v, \bar{B}\right)$, the offer will be rejected and therefore, $\alpha_{p}$ finally obtains

$$
v\left(\left\{\alpha_{p}\right\}\right)-\sum_{i \in B_{p} \backslash\left\{\alpha_{p}\right\}}\left[M S_{i}(N, v, \mathcal{B})-M S_{i}\left(B_{p} \backslash\left\{\alpha_{p}\right\}, v, \bar{B}\right)\right] .
$$

By Proposition 4.1, this payoff is not greater than $M S_{\alpha_{p}}(N, v, \mathcal{B})$, saying that the proposer $\alpha_{p}$ can not improve. If $\alpha_{p}$ offers at least $M S_{i}\left(B_{p} \backslash\left\{\alpha_{p}\right\}, v, \bar{B}\right)$ to any player $i \in B_{p} \backslash\left\{\alpha_{p}\right\}$, the offer will be accepted and $\alpha_{p}$ gets at $\operatorname{most} M S_{i}\left(B_{p} \backslash\left\{\alpha_{p}\right\}, v, \bar{B}\right)$.

Stage 1. We first get that $B_{i}=0$ for any $i \in B_{p}$ since

$$
\begin{aligned}
B_{i}= & \sum_{j \in B_{p} \backslash\{i\}}\left(b_{j}^{i}-b_{i}^{j}\right) \\
= & \sum_{j \in B_{p} \backslash\{i\}}\left[M S_{j}(N, v, \mathcal{B})-M S_{j}\left(B_{p} \backslash\{i\}, v, \bar{B}\right)\right] \\
& -\sum_{j \in B_{p} \backslash\{i\}}\left[M S_{i}(N, v, \mathcal{B})-M S_{i}\left(B_{p} \backslash\{j\}, v, \bar{B}\right)\right]
\end{aligned}
$$




$$
\begin{aligned}
= & \sum_{j \in B_{p} \backslash\{i\}}\left[S h_{j}\left(B_{p}, v\right)-S h_{j}\left(B_{p} \backslash\{i\}, v\right)\right] \\
& -\sum_{j \in B_{p} \backslash\{i\}}\left[S h_{i}\left(B_{p}, v\right)-S h_{i}\left(B_{p} \backslash\{j\}, v\right)\right] \\
= & 0,
\end{aligned}
$$

where the second equality holds due to Definition 3.1 and the last equality holds because the fact that Shapley value satisfies the balanced contributions property in Myerson [10].

Assume that player $i \in B_{p}$ makes different bids and $B_{i}^{*}$ is its net bid. If $B_{i}^{*}>0$, player $i$ becomes a proposer, but he must pay extra $B_{i}^{*}$ to other players of $B_{p} \backslash\{i\}$, which leads to its final payoff lower than $M S_{i}(N, v, \mathcal{B})$. If $B_{i}^{*} \leq 0$, player $i$ cannot improve.

Therefore, the multi-step Shapley value is indeed an $S P N E$ outcome.

Next, we prove that the outcome in any $S P N E$ of the multi-step bidding mechanism is equal to the multi-step Shapley value. Let $B_{p} \in B^{1}$.

Claim 1. In any $S P N E$, all players in $B_{p} \backslash\left\{\alpha_{p}\right\}$ for any $B_{p} \in B^{1}$ accept the offer of the proposer $\alpha_{p}$ if

$$
y_{i}^{\alpha_{p}} \geq M S_{i}\left(B_{p} \backslash\left\{\alpha_{p}\right\}, v, \bar{B}\right),
$$

and reject the offer of $\alpha_{p}$ if

$$
y_{i}^{\alpha_{p}}<M S_{i}\left(B_{p} \backslash\left\{\alpha_{p}\right\}, v, \bar{B}\right) .
$$

Consider that if player $i \in B_{p} \backslash\left\{\alpha_{p}\right\}$ accepts the offer of $\alpha_{p}, i$ will receive

$$
b_{i}^{\alpha_{p}}+y_{i}^{\alpha_{p}},
$$

whereas if he rejects the offer, he will receive

$$
b_{i}^{\alpha_{p}}+M S_{i}\left(B_{p} \backslash\left\{\alpha_{p}\right\}, v, \bar{B}\right)
$$

by induction on $h$. Therefore, in any $S P N E$, it is optimal for $i \in B_{p} \backslash\left\{\alpha_{p}\right\}$ to accept the offer of $\alpha_{p}$ if

$$
y_{i}^{\alpha_{p}}>M S_{i}\left(B_{p} \backslash\left\{\alpha_{p}\right\}, v, \bar{B}\right),
$$

and $i$ will also accept the offer of $\alpha_{p}$ if

$$
y_{i}^{\alpha_{p}}=M S_{i}\left(B_{p} \backslash\left\{\alpha_{p}\right\}, v, \bar{B}\right)
$$

because of the assumption (a). Therefore, the Claim 1 holds

Claim 2. In any $S P N E$ outcome, the offer of the proposer $\alpha_{p}$ will be accepted by all other players in $B_{p}$.

Assume that the offer $y^{\alpha_{p}}$ of the proposer $\alpha_{p}$ is rejected by some player in $B_{p} \backslash\left\{\alpha_{p}\right\}$. In this case, $\alpha_{p}$ will obtain $w=v\left(\left\{\alpha_{p}\right\}\right)-\sum_{i \in B_{p} \backslash\left\{\alpha_{p}\right\}} b_{i}^{\alpha_{p}}$.

Now assume the proposer proposes the offer

$$
z^{\alpha_{p}}=M S\left(B_{p} \backslash\left\{\alpha_{p}\right\}, v, \bar{B}\right)
$$

to players in $B_{p} \backslash\left\{\alpha_{p}\right\}$, the offer $z^{\alpha_{p}}$ will be accepted by all players in $B_{p} \backslash\left\{\alpha_{p}\right\}$ by Claim 1 and then the proposer $\alpha_{p}$ will obtain the payment

$$
\tilde{w}=-\sum_{i \in B_{p} \backslash\left\{\alpha_{p}\right\}}\left[b_{i}^{\alpha_{p}}+M S_{i}\left(B_{p} \backslash\left\{\alpha_{p}\right\}, v, \bar{B}\right)\right]+M S_{B_{p}}\left(B^{1}, v, \mathcal{B}\right) .
$$


According to Proposition 4.1, we know that $w \leq \tilde{w}$. If $w<\tilde{w}$, it says that to offer a rejected offer is not an optimal strategy for the proposer. If $w=\tilde{w}$, it says that the proposer is indifferent to offering $y^{\alpha_{p}}$ or $z^{\alpha_{p}}$ and then by the assumption (b), the proposer $\alpha_{p}$ will prefer to offer $z^{\alpha_{p}}$. Therefore, each player in $B_{p} \backslash\left\{\alpha_{p}\right\}$ will accept the offer of the proposer $\alpha_{p}$ in every $S P N E$ outcome.

Claim 3. In any $S P N E$, the offer from the proposer $\alpha_{p}$ to each $i \in B_{p} \backslash\left\{\alpha_{p}\right\}$ is

$$
y_{i}^{\alpha_{p}}=M S_{i}\left(B_{p} \backslash\left\{\alpha_{p}\right\}, v, \bar{B}\right) .
$$

According to Claim 2, the proposer $\alpha_{p} \in B_{p}$ will give the offer

$$
y_{i}^{\alpha_{p}} \geq M S_{i}\left(B_{p} \backslash\left\{\alpha_{p}\right\}, v, \bar{B}\right)
$$

to player $i \in B_{p} \backslash\left\{\alpha_{p}\right\}$. If for some $j \in B^{p} \backslash\left\{\alpha_{p}\right\}$,

$$
y_{j}^{\alpha_{p}}>M S_{j}\left(B_{p} \backslash\left\{\alpha_{p}\right\}, v, \bar{B}\right),
$$

that must induce a lower payoff for the proposer because he has to pay more to others.

Claim 4. In Round 1 of any $S P N E, B_{i}=0$ for any $i \in B_{p}$.

It is easy to derive that $\sum_{i \in B_{p}} B_{i}=0$. Denote $\Omega=\left\{i \in B_{p}: B_{i}=\max _{j \in B_{p}} B_{j}\right\}$, we have that if $\Omega=B_{p}$, the result is true because $\sum_{i \in B_{p}} B_{i}=0$.

Supposing, to the contrary, that $\Omega \neq B_{p}$, it implies that $B_{i}>0$ for some $i \in \Omega$. Take some player $j \in B_{p} \backslash \Omega$ and $B_{j}=\max _{k \in B_{p} \backslash \Omega} B_{k}$. Let player $i$ change its bids by announcing $\tilde{b}_{k}^{i}=b_{k}^{i}+\delta$ for any $k \in \Omega \backslash\{i\}, \tilde{b}_{j}^{i}=b_{j}^{i}-|\Omega| \delta$, and $\tilde{b}_{k}^{i}=b_{k}^{i}$ for any $k \in B_{p} \backslash(\Omega \cup\{j\})$, where $\delta$ is a positive real number. Then, the new net bids are: for all $k \in \Omega, \tilde{B}_{k}=B_{k}-\delta, \tilde{B}_{j}=B_{j}+|\Omega| \delta$, and for all $l \in B_{p} \backslash(\Omega \cup\{j\}), \tilde{B}_{l}=B_{l}$.

Since $B_{j}<B_{i}$, we can find a $\delta>0$ which is small enough such that $B_{j}+|\Omega| \delta<B_{i}-\delta$. Further, $\Omega$ does not change because $\tilde{\Omega}=\left\{k \in B_{p}: \tilde{B}_{k}=\max _{l \in B_{p}} \tilde{B}_{l}\right\}=\Omega$. Each player in $\Omega$ has the equal probability to be chosen as the proposer. So, by making the new bid $\tilde{b}^{i}$, if player $i$ is not the proposer, then his payoff does not change and if player $i$ is the proposer, then he will receive $\delta$ units more than making the bid $b^{i}$.

Claim 5. In Round 1 of any $S P N E$, the payoff of each player $i \in B_{p}$ is the same regardless of who is chosen as the proposer.

By Claim 4, we know that $B_{i}=0$ for all $i \in B_{p}$ in any $S P N E$. However, in order (not) to be a proposer, player $i$ have to slightly increase (decrease) its bids to others, which is impossible in an SPNE.

Claim 6. In any $S P N E$ outcome, the final payment of each player coincides with its multi-step Shapley value.

By Claims 1-3, we know that if $\left|B_{p}\right|=1$, each player $i \in B_{p}$ obtains the final payoff $M S_{B_{i}^{1}}\left(B^{1}, v, \mathcal{B}\right)=$ $M S_{i}(N, v, \mathcal{B})$. If $\left|B_{p}\right|>1$ and player $i \in B_{p}$ is the proposer in Round 1, it will obtain the final payoff $-\sum_{j \in B_{p} \backslash\{i\}}\left[b_{j}^{i}+M S_{j}\left(B_{p} \backslash\{i\}, v, \bar{B}\right)\right]+M S_{B_{p}}\left(B^{1}, v, \mathcal{B}\right)$. If $\left|B_{p}\right|>1$ and $j \neq i$ is the proposer, the final payoff of $i$ is $b_{i}^{j}+M S_{i}\left(B_{p} \backslash\{j\}, v, \bar{B}\right)$.

Denote by $p_{i}(N, v, \mathcal{B})$ the final payoff of player $i \in B_{p} \in B^{1}$ in an $S P N E$. Since in an $S P N E$, player $i$ is indifferent to all possible choices of proposers, by Claim 5 we have that

$$
\begin{aligned}
\left|B_{p}\right| p_{i}(N, v, \mathcal{B})= & -\sum_{j \in B_{p} \backslash\{i\}}\left[b_{j}^{i}+M S_{j}\left(B_{p} \backslash\{i\}, v, \bar{B}\right)\right]+M S_{B_{p}}\left(B^{1}, v, \mathcal{B}\right)+ \\
& +\sum_{j \in B_{p} \backslash\{i\}}\left[b_{i}^{j}+M S_{i}\left(B_{p} \backslash\{j\}, v, \bar{B}\right)\right] \\
= & -\sum_{j \in B_{p} \backslash\{i\}} M S_{j}\left(B_{p} \backslash\{i\}, v, \bar{B}\right)+M S_{B_{p}}\left(B^{1}, v, \mathcal{B}\right)+
\end{aligned}
$$




$$
\begin{aligned}
& +\sum_{j \in B_{p} \backslash\{i\}} M S_{i}\left(B_{p} \backslash\{j\}, v, \bar{B}\right) \\
= & \sum_{j \in B_{p} \backslash\{i\}}\left[M S_{i}\left(B_{p} \backslash\{j\}, v, \bar{B}\right)-M S_{j}\left(B_{p} \backslash\{i\}, v, \bar{B}\right)\right]+ \\
& +\sum_{i \in B_{p}} M S_{i}(N, v, \mathcal{B}) \\
= & \sum_{j \in B_{p} \backslash\{i\}}\left[M S_{i}\left(B_{p}, v, \bar{B}\right)-M S_{j}\left(B_{p}, v, \bar{B}\right)\right]+\sum_{i \in B_{p}} M S_{i}(N, v, \mathcal{B}) \\
= & \sum_{j \in B_{p} \backslash\{i\}}\left[M S_{i}(N, v, \mathcal{B})-M S_{j}(N, v, \mathcal{B})+\sum_{i \in B_{p}} M S_{i}(N, v, \mathcal{B})\right. \\
= & \left|B_{p}\right| M S_{i}(N, v, \mathcal{B}) .
\end{aligned}
$$

The penultimate and antepenultimate equations hold because of balanced contributions [10] of the Shapley value and internal surplus equality.

Therefore, $p_{i}(N, v, \mathcal{B})=M S_{i}(N, v, \mathcal{B})$.

\section{Conclusions}

In this paper, we first define and axiomatize multi-step Shapley values for cooperative games with levels structures by extending the two-step Shapley value for cooperative games with coalition structures. Second, we develop a non-cooperative mechanism to achieve the multi-step Shapley values for superadditive games, which is different from the implementation of the two-step Shapley value in Kamijo [8] when the levels structure has the degree 2.

Acknowledgements. The research has been supported by the Science Foundation of Henan University of Technology (Grant No. 2019BS049) and the Innovative Funds Plan of Henan University of Technology (Grant No. 2020ZKCJ08).

\section{REFERENCES}

[1] R.J. Aumann and J.H. Dreze, Cooperative games with coalition structures. Int. J. Game Theory 3 (1974) $217-237$.

[2] E. Calvo and E. Gutiérrez, Solidarity in games with a coalition structure. Math. Social Sci. 60 (2010) $196-203$.

[3] S. Hart and A. Mas-Colell, Bargaining and value. Econometrica 64 (1996) 357-380.

[4] R. Joosten, Dynamics, equilibria and values. dissertation, Maastricht University, The Netherlands (1996).

[5] Y. Ju, P. Borm and P. Ruys, The consensus value: a new solution concept for cooperative games. Social Choice Welfare 28 (2007) 685-703.

[6] Y. Ju and D. Wettstein, Implementing cooperative solution concepts: a generalized bidding approach. Econ. Theory 39 (2009) 307-330.

[7] Y. Kamijo, A two-step Shapley value for cooperative games with coalition structures. Int. Game Theory Rev. 11 (2009) 207-214.

[8] Y. Kamijo, The collective value: a new solution for games with coalition structures. Top 21 (2013) 572-589.

[9] B. Moldovanu and E. Winter, Core Implementation and increasing returns to scale for cooperation. J. Math. Econ. 23 (1994) 533-548.

[10] R.B. Myerson, Conference structures and fair allocation rules. Int. J. Game Theory 9 (1980) 169-182.

[11] G. Owen, Values of games with a priori unions, edited by R. Henn and O. Moeschlin. In: Essays Math. Econ. Game Theory (1977) 76-88.

[12] D. Pérez-Castrillo and D. Wettstein, Bidding for the surplus: a non-cooperative approach to the Shapley value. J. Econ. Theory 100 (2001) 274-294.

[13] L.S. Shapley, A value for $n$-person games, edited by H. Kuhn and A. Tucker. In: Contributions to the Theory of Games. Princeton University Press, 28 (1953) 307-317.

[14] R. van den Brink and Y. Funaki, Implementation and axiomatization of discounted Shapley values. Social Choice Welfare 45 (2015) 329-344.

[15] R. van den Brink, Y. Funaki and Y. Ju, Reconciling marginalism with egalitarianism: Consistency, monotonicity, and implementation of egalitarian Shapley values. Social Choice Welfare 40 (2013) 693-714. 
[16] J. Vidal-Puga and G. Bergantiños, An implementation of the Owen value. Games Econ. Behav. 44 (2003) $412-427$.

[17] J. Vidal-Puga, Implementation of the levels structure value. Ann. Oper. Res. 137 (2005) 191-209.

[18] E. Winter, A value for cooperative games with levels structure of cooperation. Int. J. Game Theory 18 (1989) $227-240$.

[19] H.P. Young, Monotonic solutions of cooperative games. Int. J. Game Theory, 14 (1985) 65-72. 